\title{
Exploration of the molecular mechanisms of cervical cancer based on mRNA expression profiles and predicted microRNA interactions
}

\author{
LIANG ZHAO ${ }^{1,2}$, ZHECHAO ZHANG ${ }^{1,3}$, HONGYAN LOU ${ }^{1,3}$, JINGJING LIANG ${ }^{1,3}$, \\ XIAOJIAN YAN ${ }^{1,3}$, WENFENG LI ${ }^{1,4}$, YUNSHENG XU ${ }^{1,5}$ and RONGYING OU ${ }^{1,3}$ \\ ${ }^{1}$ Laboratory for Advanced Interdisciplinary Research, Institute of Translational Medicine; ${ }^{2}$ Division of PET/CT, \\ Department of Radiology; Departments of ${ }^{3}$ Gynaecology and Obstetrics, ${ }^{4}$ Radiation Oncology and ${ }^{5}$ Dermatovenereology, \\ The First Affiliated Hospital of Wenzhou Medical University, Wenzhou, Zhejiang 325000, P.R. China
}

Received January 25, 2017; Accepted October 24, 2017

DOI: $10.3892 / \mathrm{ol} .2018 .8494$

\begin{abstract}
The molecular mechanisms of cervical cancer have been minimally explored with multi-omics data. In the present study, mRNA expression profiles were analyzed and combined with predicted miRNA interactions to contribute to the characterization of the underlying regulatory mechanisms of cervical cancer. A total of 92 significantly differentially expressed genes (DEGs) were identified in 33 tumor samples by comparison with 29 normal samples. mRNA-miRNA interaction network analysis revealed that 16 out of the 92 DEGs, including checkpoint kinase 1 (CHEK1), SRY-box 17 (SOX17), centrosomal protein 55, cyclin dependent kinase inhibitor $2 \mathrm{~A}$ $(C D K N 2 A)$, and inhibitor of DNA binding 4 , were the targets of 4 miRNAs which were previously reported to be involved in the regulation of cervical cancer. Tumor and normal samples could be distinctly classified into two groups based on the expression of the 16 DEGs. Furthermore, survival analysis using the SurvExpress database indicated that the 16 DEGs could individually significantly differentiate low- and high-risk cervical cancer groups. Overall, multiple biological processes are likely to participate in the progression of cervical cancer based on the pathway and function enrichment identified for the DEGs. The dysregulation of SOX17 is associated with the regulation of embryonic development, the determination of cell fate and likely promotes cancer cell transformation. The dysregulation of $C H E K 1$ and $C D K N 2 A$ further promote cancer
\end{abstract}

Correspondence to: Dr Rongying Ou or Dr Yunsheng Xu, Laboratory for Advanced Interdisciplinary Research, Institute of Translational Medicine, The First Affiliated Hospital of Wenzhou Medical University, 2 Fuxue Lane, Wenzhou, Zhejiang 325000, P.R. China

E-mail: ourongying@163.com

E-mail: xuyunsheng1018@163.com

Key words: cervical cancer, differentially expressed genes, microRNA, survival analysis cell proliferation by affecting the cell cycle checkpoint in response to DNA damage. The identification of critical genes and biological processes associated with cervical cancer may be beneficial for the exploration of the molecular mechanisms.

\section{Introduction}

Cervical cancer is one of most common types of cancer; there are $\sim 70,000$ new cases and 233,000 associated mortalities per year worldwide $(1,2)$. The reported five-year survival rate is $68 \%$ in the United States (3) and patient outcomes depend on how early the cancer is detected (4). The occurrence rate is high, at $\sim 70 \%$ in developing countries (5). In developed countries, the rate has been markedly reduced with the application of cervical screening programs (5). The greatest risk factor for cervical cancer is type 16 and 18 human papillomavirus (HPV) infection, which accounts for $75 \%$ of cervical cancer cases (6). Smoking is the next most significantly associated risk factor for cervical cancer; the incidence of invasive cervical cancer is 2-3 times higher in current or former smokers among HPV-infected women (7).

HPV infection is one of the most important causative molecular mechanisms for cervical cancer (8). The high-risk HPV types encode two oncoproteins, E6 and E7, which can inactivate tumor suppressor proteins and abrogate apoptosis (8). Using advanced microarray and next-generation sequencing technologies, researchers have explored the gene expression profile and molecular mechanisms of cervical cancer. In 2006, Wong et al (9) used oligonucleotide microarray analysis and reverse transcription-polymerase chain reaction to demonstrate that secreted phosphoprotein 1 , cyclin dependent kinase inhibitor $2 \mathrm{~A}(C D K N 2 A)$, ribosomal protein $L 39$ and Clorf10 were differentially regulated in cervical cancer compared with normal cervix tissue. Connective tissue growth factor and regulator of $\mathrm{G}$ protein signaling 1 were identified as upregulated in late stage cervical cancer (9). The pelvic lymph node metastasis ability of early-stage cervical cancer has been associated with barrier to autointegration factor 1, La ribonucleoprotein domain family member 7, Secretory carrier membrane protein, CUE domain 
containing 1 and phosphatidylethanolamine binding protein 1 by comparing gene expression profiles of tumor samples from patients with and without metastasis (10).

Several critical microRNAs (miRs/miRNAs) have also been identified in cervical cancer. Hu et al (11) indicated that miR-200a and miR-9 can predict patient survival, and that miR-200 likely affects the metastatic process of cervical cancer cells by suppressing the genes controlling cell motility. The expression of miR-424 has been demonstrated as significantly downregulated in 147 cervical cancer tissues vs. 74 normal tissues (12). miR-424 has also been identified as a crucial tumor suppressor through the mechanism of upregulating the expression and phosphorylation of CHEK1 (12).

Although various studies have explored the expression profiles of cervical cancer, a combination of mRNA and miRNA expression profiles has rarely been studied in cervical cancer. A large number of microarray expression datasets are publicly available in the Gene Expression Omnibus (GEO) database, and data-mining the deposited datasets using bioinformatics methods may advance the understanding of cervical cancer (13). In the present study, the significantly differentially expressed genes (DEGs) in cervical cancer were first identified based on two expression datasets from independent labs. DEGs were then subjected to functional annotation based on the Gene Ontology (GO) and Kyoto Encyclopedia of Genes and Genomes (KEGG) databases. The regulation mechanisms between the identified DEGs and reported miRNAs in cervical cancer were then explored. The prognostic performance of the identified DEGs were virtually validated using the SurvExpress online database.

\section{Materials and methods}

mRNA and miRNA expression profiles. Datasets for cervical cancer were obtained from the publicly available GEO database (http://www.ncbi.nlm.nih.gov/geo/). Two mRNA expression profile datasets that used the same platform and contained normal tissue controls were selected. GSE63678 was submitted by Pappa et al in 2014 (14), and contains 5 normal tissues and 5 cancer tissues, whereas GSE63514 was submitted by den Boon et al in 2014 (15) and includes 24 normal tissue and 28 cancer tissue expression profiles. Both of these datasets had been produced using the Affymetrix Human Genome U133 Plus 2.0 array. Further information regarding the original samples and experiments are documented in the referenced manuscripts.

Identification of differentially expressed genes. An R script and database produced in-house were used for the data analysis and annotation. In brief, mRNA expression profiles underwent background correction, normalization and $\log _{2}$ transformation with the GeneChip Robust Multi-array Analysis algorithm (16). Control probe sets were filtered out, and the mean expression was calculated for genes with multiple probes. Finally, the Linear Models for Microarray Analysis algorithm in Bioconductor was applied for DEG screening (17). The common DEGs between the two data sets were also identified. Criteria to indicate a statistically significant difference were set to $\mathrm{P} \leq 0.05$ and absolute $\log _{2}$ (fold-change) $\geq 2$.
Table I. Top 20 differentially expressed genes in the GSE63514 and GSE63678 datasets.

\begin{tabular}{lcc}
\hline & \multicolumn{2}{c}{ Fold-change } \\
\cline { 2 - 3 } Gene & GSE63514 & GSE63678 \\
\hline Clorf116 & -2.57 & 2.17 \\
CEACAM6 & -2.05 & 3.32 \\
BIRC5 & 2.10 & 2.14 \\
CCNB1 & 2.11 & 2.45 \\
CENPF & 2.27 & 2.18 \\
AURKA & 2.31 & 2.43 \\
BUB1B & 2.37 & 2.06 \\
CDKN3 & 2.41 & 2.20 \\
CHEK1 & 2.44 & 2.12 \\
CEP55 & 2.50 & 2.51 \\
ASPM & 2.55 & 2.77 \\
APOBEC3B & 2.58 & 2.06 \\
CENPN & 2.63 & 2.02 \\
CDK1 & 2.68 & 2.35 \\
CCNE2 & 2.75 & 2.56 \\
CENPE & 2.98 & 2.06 \\
CDC7 & 3.00 & 2.54 \\
ATAD2 & 3.59 & 2.36 \\
CA9 & 4.19 & 2.39 \\
CDKN2A & 6.41 & 2.46 \\
\hline & &
\end{tabular}

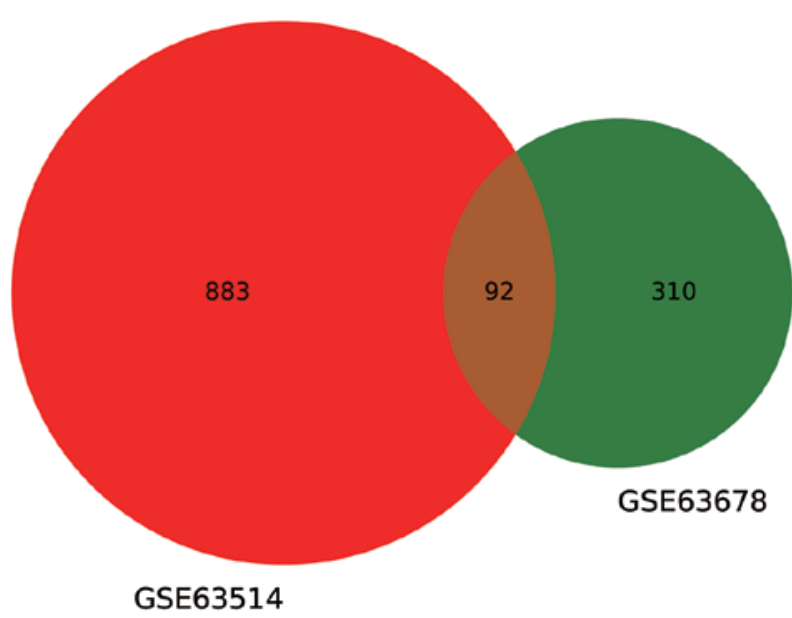

Figure 1. Venn diagram illustrating the differentially expressed genes identified in GSE63514 and GSE63678, and those common between the datasets.

GO and KEGG pathway annotation. The identified DEGs were subjected to GO and KEGG pathway enrichment analysis using the Database for Annotation, Visualization and Integrated Discovery (DAVID) online (18). GO terms were identified in the Biological Process (BP), Cellular Component (CC) and Molecular Function (MF) categories. $\mathrm{P}<0.05$ was set as the significance threshold.

miRNAs may be critical in carcinogenesis and metastasis through their regulation of mRNA expression; by searching PubMed, 6 critical miRNAs involved in the 
Table II. Kyoto Encyclopedia of Genes and Genomes pathway enrichment results for the common differentially expressed genes.

\begin{tabular}{|c|c|c|}
\hline Term & P-value & Genes \\
\hline hsa04110: cell cycle & $9.02 \times 10^{-9}$ & $\begin{array}{l}C D K N 2 A, C D C 7, M C M 2, C D K 1, C C N E 2, C H E K 1, B U B 1 B, C C N B 1, \\
M A D 2 L 1, M C M 4\end{array}$ \\
\hline hsa04115: p53 signaling pathway & $2.20 \times 10^{-5}$ & CDKN2A, CDK1, CCNE2, RRM2, CHEK1, CCNB1 \\
\hline hsa04114: oocyte meiosis & 0.00234 & $C D K 1, A U R K A, C C N E 2, M A D 2 L 1, P G R$ \\
\hline
\end{tabular}

Table III. Top 5 GO terms for the common differentially expressed genes.

A, Molecular function

\begin{tabular}{llll}
\hline ID & \multicolumn{1}{c}{ GO Term } & P-value & \multicolumn{1}{c}{ Genes } \\
\hline GO:0008017 & microtubule binding & $9.49 \times 10^{-5}$ & KIF4A, PRC1, CENPE, KIF 11, KIF20A, BIRC5, CRYAB, NUSAP1 \\
GO:0005524 & ATP binding & $1.72 \times 10^{-4}$ & ATAD2, KIF4A, CDC7, OAS2, FGFR2 \\
GO:0005515 & protein binding & $5.28 \times 10^{-4}$ & $C D K N 2 A, D T L, K I F 4 A, U B D, P G R$ \\
GO:0019901 & protein kinase binding & $7.13 \times 10^{-4}$ & $C D K N 2 A, P R C 1, A U R K A, F O X M 1, C C N E 2, K I F 11, K I F 20 A$, \\
& & & $C C N B 1, K R T 17$ \\
GO:0003777 & microtubule motor activity & $7.33 \times 10^{-4}$ & KIF4A, CENPE, KIF $2 C, K I F 11, K I F 20 A$ \\
\hline
\end{tabular}

B, Biological process

\begin{tabular}{|c|c|c|c|}
\hline ID & GO Term & P-value & Genes \\
\hline GO:0000082 & $\begin{array}{l}\text { G1/S transition of } \\
\text { mitotic cell cycle }\end{array}$ & $8.32 \times 10^{-11}$ & $C D K N 2 A, C D C 7, M C M 2, C D K N 3, I D 4$ \\
\hline GO:0051301 & cell division & $1.66 \times 10^{-9}$ & $C D C 7, S M C 4, C D K 1, C C N B 1, M A D 2 L 1$ \\
\hline GO:0007067 & mitotic nuclear division & $4.57 \times 10^{-8}$ & CDK1, AURKA, NEK2, CEP55, BIRC5 \\
\hline GO:0000086 & $\begin{array}{l}\mathrm{G} 2 / \mathrm{M} \text { transition of } \\
\text { mitotic cell cycle }\end{array}$ & $4.38 \times 10^{-7}$ & $\begin{array}{l}\text { MELK, CDK1, HMMR, AURKA, NEK2, FOXM1, CHEK1, } \\
\text { BIRC5, CCNB1 }\end{array}$ \\
\hline GO:0007062 & sister chromatid cohesion & $7.67 \times 10^{-7}$ & $\begin{array}{l}\text { CENPE, CENPN, CENPF, SLC35F6, KIF2C, BUB1B, BIRC5, } \\
\text { MAD2L1 }\end{array}$ \\
\hline
\end{tabular}

C, Cellular component

\begin{tabular}{llll}
\hline ID & \multicolumn{1}{c}{ GO Term } & P-value & \multicolumn{1}{c}{ Genes } \\
\hline GO:0030496 & midbody & $2.49 \times 10^{-11}$ & KIF4A, ECT2, PRC1, CEP55, BIRC5 \\
GO:0005654 & nucleoplasm & $3.29 \times 10^{-11}$ & $A T A D 2, C D K N 2 A, D T L, S O X 17, P G R$ \\
GO:0005634 & nucleus & $9.58 \times 10^{-10}$ & $A T A D 2, C D K N 2 A, D T L, S O X 17, P G R$ \\
GO:0000775 & chromosome, & $8.95 \times 10^{-9}$ & CENPE, CENPN, MKI67, CENPF, SLC35F6, KIF2C, OIP5, \\
& centromeric region & & BIRC5 \\
GO:0005876 & spindle microtubule & $5.70 \times 10^{-8}$ & KIF4A, PRC1, CDK1, AURKA, KIF11, BIRC5, NUSAP1 \\
\hline
\end{tabular}

GO, Gene Ontology; ATP, adenosine triphosphate.

development of cervical cancer were identified: $\operatorname{miR}-200 a-5 p$, miR-9-5p, miR-424-5p, miR-133b, miR-224-3p and miR-506-5p (11,12,19-21). A regulation network between the common DEGs and 4 miRNAs was constructed. miRNA targets were predicted based on the microcosm (22), mirTarbase (23) and TargetScan (24) databases, and the associations between the common DEGs and the target genes were identified. Finally, the regulation network was plotted using CyTargetLinker plugin (25) in Cytoscape v3.5.1 (26).

Co-expression and interaction network analysis. The concept of co-expression can be used for the identification of novel mechanisms that contribute to tumorigenesis and progression (27). To identify patterns of co-expression, the odds 
ratio (OR) between each pair of query genes was calculated, and significant pairs were selected based on the cervical carcinoma data (28) from The Cancer Genome Atlas (TCGA) (28) using cBioPortal (29). The interactions between the identified DEGs and potential cancer drug targets were predicted based on various databases, including Reactome (30), DrugBank (31), CancerRxGene (32) and PANTHER (33).

In-silico validation. To validate the clinical significance of the identified DEGs, they were first validated in large cohort cervical cancer samples from TCGA (28) using cBioPortal (30). The prognostic performance of the 16 selected DEGs was then virtually evaluated using the SurvExpress database, which includes gene expression datasets with clinical outcome data (34). One cervical cancer dataset (28), including clinical information, was selected from TCGA for virtual validation. Detailed information regarding the dataset can be found in the original study (28). Parameters were carefully selected according to the developer's guide that provides optimized parameters (34). A heatmap for all samples, based on the miRNA target genes, was plotted using the heatmap module in Bioconductor (35).

\section{Results}

DEGs in cervical cancer. Subsequent to background correction and normalization, the median gene expression values for the two datasets were similar (data not shown). A total of 975 and 402 DEGs were screened in GSE63514 and GSE63678, respectively. In GSE63514, this included 523 upregulated (53.6\%) and 452 downregulated genes $(46.4 \%)$, and in GSE63678, 160 upregulated (39.8\%) and 242 downregulated genes (60.2\%). Fig. 1 illustrates that 92 common genes were differentially expressed between GSE63514 and GSE63678, and fold-changes for the top 20 up/downregulated common DEGs are listed in Table I.

GO and KEGG pathway annotation. To identify the biological functions of the common DEGs, GO and KEGG pathway enrichment analysis were performed using the DAVID online tool. The results indicate that the common DEGs are significantly enriched in three KEGG pathways (Table II). A total of 10 genes were associated with 'cell cycle' $\left.\mathrm{P}=9 \times 10^{-9}\right)$, 6 with 'p53 signaling' $\left(\mathrm{P}=2.2 \times 10^{-5}\right)$ and 5 genes with 'oocyte meiosis' ( $\mathrm{P}=0.002)$. In $\mathrm{GO}$ terms, $\mathrm{DEGs}$ were most commonly associated with molecular functions of 'microtubule binding' $\left(\mathrm{P}=9.4 \times 10^{-5}\right)$, 'ATP binding' $\left(\mathrm{P}=1.7 \times 10^{-4}\right)$ and 'protein binding' $\left(\mathrm{P}=5.2 \times 10^{-4}\right.$; Table III). The top 5 most significantly enriched terms in biological process were ' $\mathrm{S}$ transition of mitotic cell cycle' $\left(\mathrm{P}=8.3 \times 10^{-11}\right)$, 'cell division' $\left(\mathrm{P}=1.6 \times 10^{-9}\right)$, 'mitotic nuclear division' $\left(\mathrm{P}=4.5 \times 10^{-8}\right)$, ' $\mathrm{G} 2 / \mathrm{M}$ transition of mitotic cell cycle' $\left(\mathrm{P}=4.3 \times 10^{-7}\right)$ and 'sister chromatid cohesion' $\left(\mathrm{P}=7.6 \times 10^{-7}\right.$; Table III). The top 5 significantly enriched terms in cellular component were 'midbody' $\left(\mathrm{P}=2.4 \times 10^{-11}\right)$, 'nucleoplasm' $\left(\mathrm{P}=3.2 \times 10^{-11}\right)$, 'nucleus' $\left(\mathrm{P}=9.5 \times 10^{-10}\right)$, 'chromosome centromeric region' $\left(\mathrm{P}=8.9 \times 10^{-9}\right)$ and 'spindle microtubule' $\left(\mathrm{P}=5.7 \times 10^{-8}\right.$; Table III $)$.

mRNA-miRNA network construction. The 6 identified miRNAs could target 231, 2,792 and 3,143 genes in the
Table IV. The significant gene co-expression pairs among the differentially expressed genes.

\begin{tabular}{llcc}
\hline Gene A & Gene B & P-value & Log odds ratio \\
\hline SOX17 & PEG3 & $<0.001$ & $>3$ \\
SOX17 & GREB1 & $<0.001$ & $>3$ \\
SOX17 & ID4 & $<0.001$ & $>3$ \\
SOX17 & SPON1 & $<0.001$ & $>3$ \\
PEG3 & GREB1 & $<0.001$ & $>3$ \\
PEG3 & ID4 & 0.023 & 2.759 \\
GREB1 & ID4 & 0.007 & 2.521 \\
CDKN2A & OIP5 & 0.011 & 1.858 \\
KIF2C & CCNE2 & 0.004 & 1.709 \\
CDKN2A & APOBEC3B & 0.030 & 1.475 \\
CCNE2 & PRC1 & 0.035 & 1.243 \\
CHEK1 & APOBEC3B & 0.041 & 1.208 \\
\hline
\end{tabular}

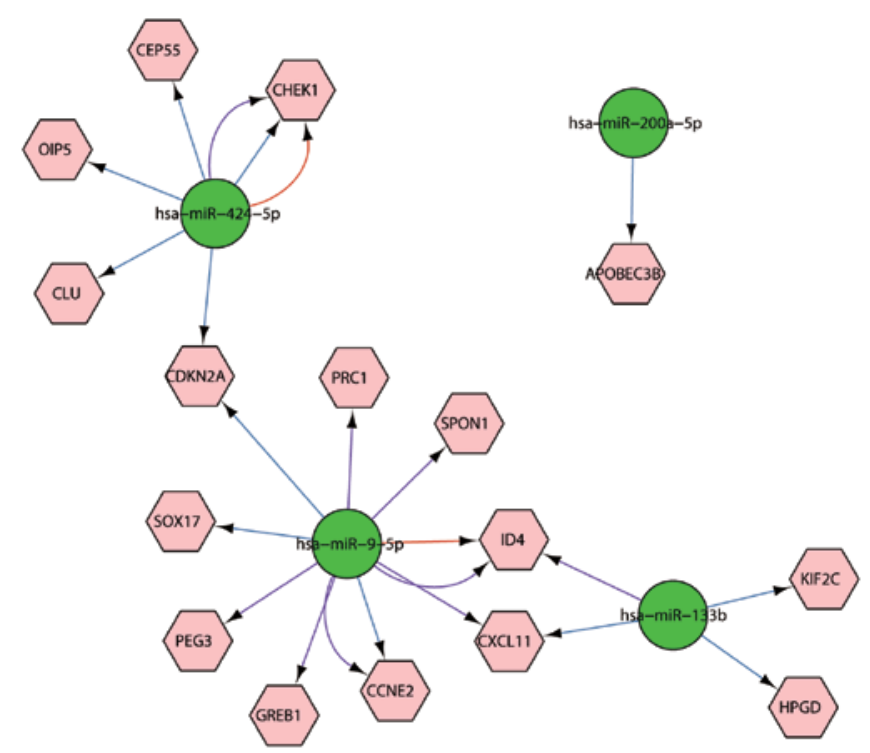

Figure 2. Regulation network of the 16 common deferentially expressed genes (pink hexagons) and the 4 documented microRNAs (green circles). The edge color represents the interaction information source. Red, miRTarBase; blue, MicroCosm; purple, TargetScan.

miRTarBase, MicroCosm and TargetScan databases, respectively. Among the target genes, 16 genes were common DEGs. Based on the interaction network between the 16 genes and 4 miRNAs (Fig. 2), it was identified that miR-424-5p could regulate 5 genes, miR-9-5p could regulate 9 genes, miR-133b could regulate 4 genes and miR-200a-5p could regulate one gene. All samples from the two datasets were subjected to hierarchical clustering using the expression of the 16 DEGs. Fig. 3 illustrates that tumor (red) and normal (blue) samples can almost be classified into two groups. Data for a number of tumor and normal samples were confounded; this was most likely due to tumor heterogeneity or expression value variation.

Co-expression and interaction network analysis. To reveal gene co-expression patterns, the significant gene pairings were 

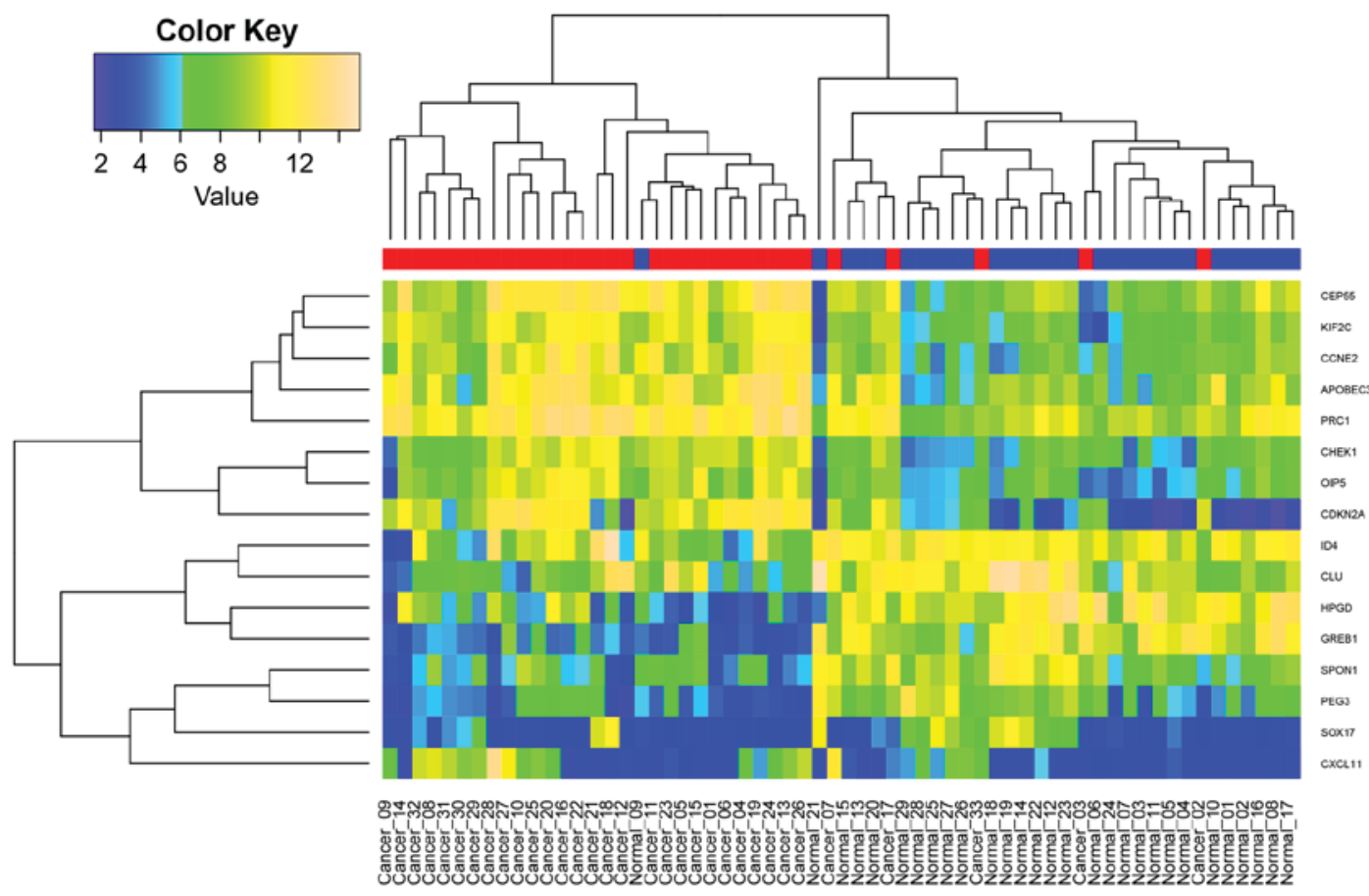

Figure 3. Heat map illustrating the different expression patterns of the 16 differentially expressed genes in all samples. The x-axis represents individual samples and the y-axis represents genes. The top bar indicates the tumor samples (red) and the normal samples (blue).

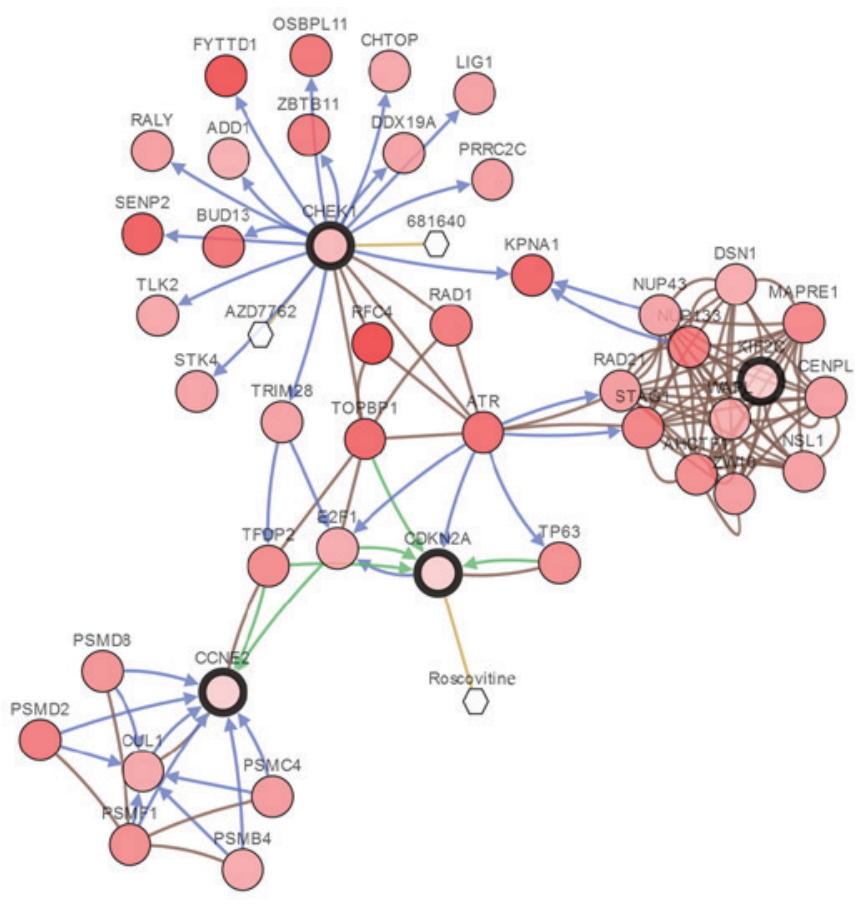

Figure 4. Interaction network between the identified differentially expressed genes and potential drugs. Each node is color coded along a white to red color gradient, indicating the total frequency of alteration across the selected case set, the deeper the red, the higher the frequency of alteration). The hexagons represent a drug and the circles represent a gene. Node border: Thin, linker nodes or targets of DEGs; thick, selected DEGs. Edge colors: Blue, controls state change of; brown, controls transport of; green, controls expression of; yellow, controls phosphorylation of. DEGs, differentially expressed genes.

selected based on odds ratio. This analysis identified 12 gene pairings among the 16 DEGs. SRY-box 17 (SOX17) tended to be co-expressed with paternally expressed 3 (PEG3), growth regulation by estrogen in breast cancer 1 (GREB1), inhibitor of DNA binding 4 (ID4) and Spondin 1 (SPONI), respectively $(\mathrm{P}<0.01$; Table IV). PEG3 was often co-expressed with ID4 and GREB1 $(\mathrm{P}<0.05$; Table IV). In addition, the interaction network indicated that Checkpoint kinase 1 (CHEKI), Cyclin E2 (CCNE2), CDKN2A and Wings apart-like protein homolog interact via Transcription factor Dp-2, E2F transcription factor 1, Topoisomerase (DNA) II binding protein 1, ATR and karyopherin subunit $\alpha 1$ (Fig. 4). Cancer drugs Roscovitine, AZD7762 and 681640 can target CDKN2A and CHEK1 respectively (Fig. 4).

In-silico validation. In a large cohort of cervical cancer samples, the identified common DEGs were all differentially expressed (z-score, $<-2$ or $>2$ ). The percentage of sample ranged from 1.3 to $12 \%$ (Fig. 5). The prognostic performance of the 16 genes was virtually validated using the SurvExpress online tool's cervical cancer dataset. This analysis indicated that low- and high-risk cervical cancer groups could be significantly differentiated based on the 16 genes (Fig. 6). The low P-value ( $\mathrm{P}=0.001)$ and high concordance index suggested that an accurate prediction of prognosis could be achieved based on the DEGs.

\section{Discussion}

With the rapid development of microarray and NGS technologies, more and more cervical cancer molecular biomarkers have been identified. However, there has been limited integration of multi-omic data for the exploration of the molecular mechanisms of cervical cancer. In this study, mRNA expression profiles and previously identified miRNA-mRNA interactions were integrated to contribute to the characterization of the complex mechanism of cervical cancer. Systematic analysis revealed that 92 genes were simultaneously differentially 


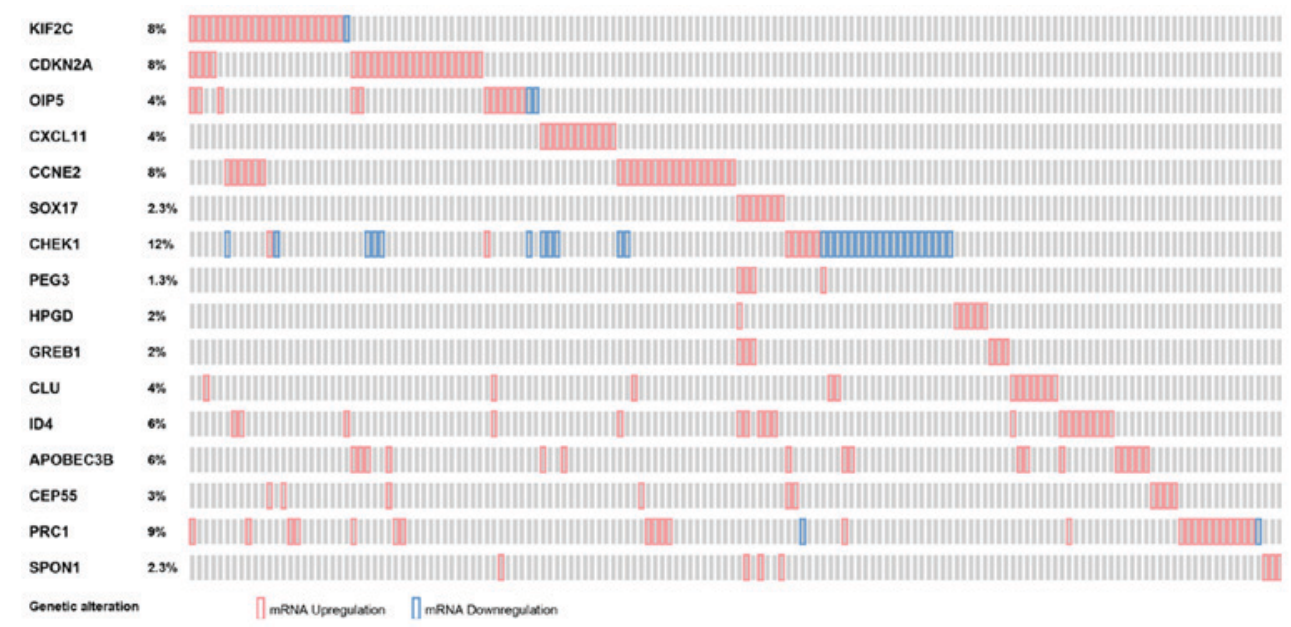

Figure 5. Expression of the identified differentially expressed genes in large cohorts of cervical cancer samples.

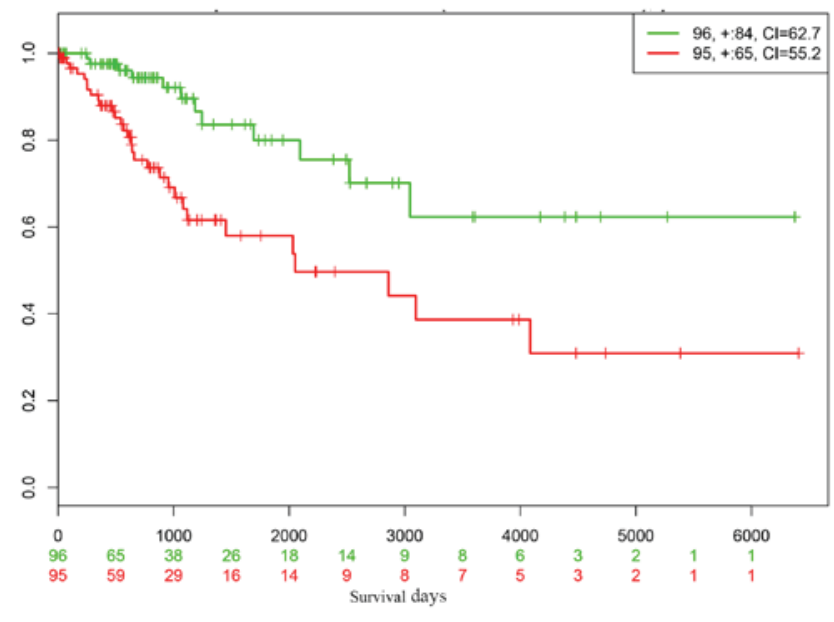

Figure 6. Kaplan-Meier curves for high- and low-risk groups, represented with red and green curves respectively, of a cervical cancer dataset in the SurvExpress database. Censored samples are marked with ' + '. The red and green numbers on the $\mathrm{x}$-axis represent the number of mortalities of high- and low-risk individuals, respectively, occurring prior to the end of follow-up. The number of samples, censored number and CI are displayed in the top-right insets. The red and green numbers below the $\mathrm{x}$-axis represent the number of low- and high-risk patients alive. CI, concordance index.

expressed in 33 tumor tissues and that 16 DEGs may have been regulated by 4 critical miRNAs. The tumor and normal tissues were clearly classified into two groups based on the expression of the 16 DEGs.

Pathway enrichment analysis indicated that 6 DEGs were associated with 'p53 signaling'. The study by Xiao et al (36) indicated that fra- 1 was significantly downregulated in cervical cancer compared with adjacent normal tissue. fra-l can dysregulate p53 signaling via regulating the expression of p53 and MDM2 in vivo.

Based on an mRNA-miRNA interaction network, the mechanism of cervical cancer was further characterized, indicating that miR-424-5p can regulate $C H E K 1$. The protein encoded by this gene is required for checkpoint-mediated cell cycle arrest in response to DNA damage (37). Immunohistochemical study has indicated the ubiquitous expression of CHEK1 protein in cervical cancer, ovarian carcinoma and other types of cancer (38). In 2011, Mazumder et al (39) demonstrated that the deletion and methylation of CHEK1 were associated with the progression of cervical carcinoma, and that the inactivation of the ATM-CHEK1 DNA damage response pathway participated in cervical cancer. ATM is activated by phosphorylation in response to DNA damage, which in turn activates its downstream target $C H E K 1$, suggesting that inactivation of the ATM-CHEK1-associated DNA damage response pathway may have an important role in the development of cervical carcinoma (39). In addition, $\mathrm{Xu}$ et al (12) identified a critical tumor suppressive role for miR-424 in the progression of cervical cancer, at least partly via upregulating the expression of CHEK1 and p-CHEK1. Overexpression of miR-424 inhibited the expression of CHEK1 and p-CHEK1 at residue Ser345, and decreased the activity of the luciferase-reporter containing the 3'-untranslated region of CHEK1. Hsieh et al (40) revealed that Euphorbia antiquorum extracts could downregulate topoisomerase and activate ATM kinase, inducing the CHEK $1 / 2$ and mitogen activated protein kinase signaling pathways, and promoting the degradation of cell division cycle $25 \mathrm{~A}$ to induce $\mathrm{S}$-phase arrest in HeLa cells.

An additional critical gene is $C D K N 2 A$, which may be regulated by miR-424-5p or miR-9-5p. $C D K N 2 A$ encodes 2 main proteins, including p16INK4, which is a cyclin-dependent kinase inhibitor, and p14ARF, which can bind the p53-stabilizing protein MDM2 (41). These proteins participate in the regulation of two critical cell cycle regulatory pathways, the p53 pathway and the RB1 pathway. p16 can decelerate cell cycle progression from G1 phase to $\mathrm{S}$ phase. Wijetunga et al (42) reported a novel potential link between early cervical cancer disease progression and CpG-DNA methylation within the area 700 bp downstream of the transcriptional start site of $C D K N 2 A$, which may lead to increased p16INK4A/p14ARF expression prior to the development of malignant disease. In advanced cervical cancers, the majority of cells exhibited methylated $C D K N 2 A$, a lack of p16INK4A protein and no expression of HPV E7, suggesting p16INK4A inactivation may be a mechanism of blocking the cyclin D-RB1 pathway in invasive cervical cancer (43).

SOX17 encodes a member of the SOX family involved in the regulation of embryonic development and the determination 
of cell fate, and can be regulated by miR-9-5p (44). SOX17 methylation frequency has been reported to be significantly higher in squamous cell carcinoma than in cervical adenocarcinoma, indicating that $S O X 17$ silencing may contribute to the aberrant activation of Wnt signaling in cervical cancer (45). Furthermore, SOX17 exhibited general hypermethylation in $\mathrm{CpG}$ sites analyzed in cervical cancer samples and may interact directly with CTNNB1 (46). Genes previously unreported, to the best of our knowledge, including CEP55 and ID4, were also identified in this study.

In summary, the development and progression of cervical cancer is likely to be induced by various processes. The present study identified DEGs that may be potential targets for regulation in cervical cancer.

\section{Acknowledgements}

The present study was supported by grants from the National Natural Science Foundation of China (grant nos. 81373075, 8167140, 81371748 and 81571395).

\section{References}

1. Parkin DM, Bray F, Ferlay J and Pisani P: Estimating the world cancer burden: Globocan 2000. Int J Cancer 94: 153-156, 2001.

2. Bosch FX and de Sanjosé S: Chapter 1: Human papillomavirus and cervical cancer-burden and assessment of causality. J Natl Cancer Inst Monogr: 3-13, 2003.

3. Thanagumtorn K: Survival rate of recurrent cervical carcinoma. J Med Assoc Thai 95 (Suppl 3): S125-S130, 2012.

4. zur Hausen H: Papillomaviruses and cancer: From basic studies to clinical application. Nat Rev Cancer 2: 342-350, 2002.

5. Canavan TP and Doshi NR: Cervical cancer. Am Fam Physician 61: 1369-1376, 2000.

6. Bosch FX, Manos MM, Muñoz N, Sherman M, Jansen AM, Peto J, Schiffman MH, Moreno V, Kurman R and Shah KV: Prevalence of human papillomavirus in cervical cancer: A worldwide perspective. International biological study on cervical cancer (IBSCC) Study Group. J Natl Cancer Inst 87: 796-802, 1995.

7. Winkelstein W Jr: Smoking and cervical cancer-current status: A review. Am J Epidemiol 131: 945-960, 1990.

8. Ledwaba T, Dlamini Z, Naicker S and Bhoola K: Molecular genetics of human cervical cancer: Role of papillomavirus and the apoptotic cascade. Biol Chem 385: 671-682, 2004.

9. Wong YF, Cheung TH, Tsao GS, Lo KW, Yim SF, Wang VW, Heung MM, Chan SC, Chan LK, Ho TW, et al: Genome-wide gene expression profiling of cervical cancer in Hong Kong women by oligonucleotide microarray. Int J Cancer 118: 2461-2469, 2006.

10. Biewenga P, Buist MR, Moerland PD, Ver Loren van Themaat E, van Kampen AH, ten Kate FJ and Baas F: Gene expression in early stage cervical cancer. Gynecol Oncol 108: 520-526, 2008.

11. Hu X, Schwarz JK, Lewis JS Jr, Huettner PC, Rader JS, Deasy JO, Grigsby PW and Wang X: A microRNA expression signature for cervical cancer prognosis. Cancer Res 70: 1441-1448, 2010.

12. Xu J, Li Y, Wang F, Wang X, Cheng B, Ye F, Xie X, Zhou C and Lu W: Suppressed miR-424 expression via upregulation of target gene Chk1 contributes to the progression of cervical cancer. Oncogene 32: 976-987, 2013.

13. Berger B, Peng J and Singh M: Computational solutions for omics data. Nat Rev Genet 14: 333-346, 2013.

14. Pappa KI, Polyzos A, Jacob-Hirsch J, Amariglio N, Vlachos GD, Loutradis D and Anagnou NP: Profiling of discrete gynecological cancers reveals novel transcriptional modules and common features shared by other cancer types and embryonic stem cells. PLoS One 10: e0142229, 2015.

15. den Boon JA, Pyeon D, Wang SS, Horswill M, Schiffman M, Sherman M, Zuna RE, Wang Z, Hewitt SM, Pearson R, et al: Molecular transitions from papillomavirus infection to cervical precancer and cancer: Role of stromal estrogen receptor signaling. Proc Natl Acad Sci USA 112: E3255-E3264, 2015.
16. Gentleman RC, Carey VJ, Bates DM, Bolstad B, Dettling M, Dudoit S, Ellis B, Gautier L, Ge Y, Gentry J, et al: Bioconductor: Open software development for computational biology and bioinformatics. Genome Biol 5: R80, 2004.

17. Kerr MK: Linear models for microarray data analysis: Hidden similarities and differences. J Comput Biol 10: 891-901, 2003.

18. Dennis G Jr, Sherman BT, Hosack DA, Yang J, Gao W, Lane HC and Lempicki RA: DAVID: Database for annotation, visualization, and integrated discovery. Genome Biol 4: P3, 2003.

19. Qin W, Dong P, Ma C, Mitchelson K, Deng T, Zhang L, Sun Y, Feng X, Ding Y, Lu X, et al: MicroRNA-133b is a key promoter of cervical carcinoma development through the activation of the ERK and AKT1 pathways. Oncogene 31: 4067-4075, 2012.

20. Fang W, Shu S, Yongmei L, Endong Z, Lirong Y and Bei S: miR-224-3p inhibits autophagy in cervical cancer cells by targeting FIP200. Sci Rep 6: 33229, 2016.

21. Wen SY, Lin Y, Yu YQ, Cao SJ, Zhang R, Yang XM, Li J, Zhang YL, Wang YH, Ma MZ, et al: miR-506 acts as a tumor suppressor by directly targeting the hedgehog pathway transcription factor Gli3 in human cervical cancer. Oncogene 34: 717-725, 2015.

22. Griffiths-Jones S, Saini HK, van Dongen S and Enright AJ: miRBase: Tools for microRNA genomics. Nucleic Acids Res 36 (Database Issue): D154-D158, 2008

23. Chou CH, Chang NW, Shrestha S, Hsu SD, Lin YL, Lee WH, Yang CD, Hong HC, Wei TY, Tu SJ, et al: miRTarBase 2016: Updates to the experimentally validated miRNA-target interactions database. Nucleic Acids Res 44: D239-D247, 2016.

24. Lewis BP, Shih IH, Jones-Rhoades MW, Bartel DP and Burge CB: Prediction of mammalian microRNA targets. Cell 115: 787-798, 2003.

25. Kutmon M, Kelder T, Mandaviya P, Evelo CT and Coort SL: CyTargetLinker: A cytoscape app to integrate regulatory interactions in network analysis. PLoS One 8: e82160, 2013.

26. Smoot ME, Ono K, Ruscheinski J, Wang PL and Ideker T: Cytoscape 2.8: New features for data integration and network visualization. Bioinformatics 27: 431-432, 2011.

27. Ciriello G, Cerami E, Sander C and Schultz N: Mutual exclusivity analysis identifies oncogenic network modules. Genome Res 22: 398-406, 2012.

28. Cancer Genome Atlas Research Network; Albert Einstein College of Medicine; Analytical Biological Services; Barretos Cancer Hospital; Baylor College of Medicine; Beckman Research Institute of City of Hope; Buck Institute for Research on Aging; Canada's Michael Smith Genome Sciences Centre; Harvard Medical School; Helen F. Graham Cancer Center \& Research Institute at Christiana Care Health Services, et al: Integrated genomic and molecular characterization of cervical cancer. Nature 543: 378-384, 2017.

29. Gao J, Aksoy BA, Dogrusoz U, Dresdner G, Gross B, Sumer SO, Sun Y, Jacobsen A, Sinha R, Larsson E, et al: Integrative analysis of complex cancer genomics and clinical profiles using the cBioPortal. Sci Signal 6: pl1, 2013.

30. Fabregat A, Sidiropoulos K, Garapati P, Gillespie M,Hausmann K, Haw R, Jassal B, Jupe S, Korninger F, McKay S, et al: The Reactome pathway Knowledgebase. Nucleic Acids Res 44: D481-D487, 2016.

31. Law V, Knox C, Djoumbou Y, Jewison T, Guo AC, Liu Y, Maciejewski A, Arndt D, Wilson M, Neveu V, et al: DrugBank 4.0: Shedding new light on drug metabolism. Nucleic Acids Res 42 (Database Issue): D1091-D1097, 2014

32. Yang W, Soares J, Greninger P, Edelman EJ, Lightfoot H, Forbes S, Bindal N, Beare D, Smith JA, Thompson IR, et al: Genomics of drug sensitivity in cancer (GDSC): A resource for therapeutic biomarker discovery in cancer cells. Nucleic Acids Res 41 (Database Issue): D955-D961, 2013.

33. Mi H, Huang X, Muruganujan A, Tang H, Mills C, Kang D and Thomas PD: PANTHER version 11: Expanded annotation data from Gene Ontology and Reactome pathways, and data analysis tool enhancements. Nucleic Acids Res 45: D183-D189, 2017.

34. Aguirre-Gamboa R, Gomez-Rueda H, Martínez-Ledesma E, Martínez-TorteyaA,Chacolla-HuaringaR,Rodriguez-BarrientosA, Tamez-Peña JG and Treviño V: SurvExpress: An online biomarker validation tool and database for cancer gene expression data using survival analysis. PLoS One 8: e74250, 2013.

35. Hadley W: Ggplot2: Elegrant graphics for data analysis. Springer, Switzerland, 2016.

36. Xiao S, Zhou Y, Yi W, Luo G, Jiang B, Tian Q, Li Y and Xue M: Fra-1 is downregulated in cervical cancer tissues and promotes cervical cancer cell apoptosis by p53 signaling pathway in vitro. Int J Oncol 46: 1677-1684, 2015. 
37. Han X, Tang J, Wang J, Ren F, Zheng J, Gragg M, Kiser P, Park PS, Palczewski K, Yao X and Zhang Y: Conformational change of human checkpoint kinase 1 (Chk1) induced by DNA damage. J Biol Chem 291: 12951-12959, 2016.

38. Gao Q, Huang X, Tang D, Cao Y, Chen G, Lu Y, Zhuang L, Wang S, Xu G, Zhou J and Ma D: Influence of chk1 and plk1 silencing on radiation- or cisplatin-induced cytotoxicity in human malignant cells. Apoptosis 11: 1789-1800, 2006.

39. Mazumder Indra D, Mitra S, Roy A, Mondal RK, Basu PS, Roychoudhury S, Chakravarty R and Panda CK: Alterations of ATM and CADM1 in chromosomal 11q22.3-23.2 region are associated with the development of invasive cervical carcinoma. Hum Genet 130: 735-748, 2011.

40. Hsieh WT, Lin HY, Chen JH, Lin WC, Kuo YH, Wood WG, Lu HF and Chung JG: Latex of Euphorbia antiquorum-induced S-phase arrest via active ATM kinase and MAPK pathways in human cervical cancer HeLa cells. Environ Toxicol 30: $1205-1215,2015$

41. Robertson KD and Jones PA: Tissue-specific alternative splicing in the human INK4a/ARF cell cycle regulatory locus. Oncogene 18: 3810-3820, 1999.

42. Wijetunga NA, Belbin TJ, Burk RD, Whitney K, Abadi M, Greally JM, Einstein MH and Schlecht NF: Novel epigenetic changes in CDKN2A are associated with progression of cervical intraepithelial neoplasia. Gynecol Oncol 142: 566-573, 2016.
43. Nuovo GJ, Plaia TW, Belinsky SA, Baylin SB and Herman JG: In situ detection of the hypermethylation-induced inactivation of the p16 gene as an early event in oncogenesis. Proc Natl Acad Sci USA 96: 12754-12759, 1999.

44. Irie N, Weinberger L, Tang WW, Kobayashi T, Viukov S, Manor YS, Dietmann S, Hanna JH and Surani MA: SOX17 is a critical specifier of human primordial germ cell fate. Cell 160: 253-268, 2015

45. van der Meide WF, Snellenberg S, Meijer CJ, Baalbergen A, Helmerhorst TJ, van derSluis WB, Snijders PJ and Steenbergen RD: Promoter methylation analysis of $\mathrm{WNT} / \beta$-catenin signaling pathway regulators to detect adenocarcinoma or its precursor lesion of the cervix. Gynecol Oncol 123: 116-122, 2011.

46. Chen YC, Huang RL, Huang YK, Liao YP, Su PH, Wang HC, Chang CC, Lin YW, Yu MH, Chu TY and Lai HC: Methylomics analysis identifies epigenetically silenced genes and implies an activation of $\beta$-catenin signaling in cervical cancer. Int $J$ Cancer 135: 117-127, 2014.

(i) (-) This work is licensed under a Creative Commons Attribution-NonCommercial-NoDerivatives 4.0 International (CC BY-NC-ND 4.0) License. 